\title{
Incidence and Risk Factors of Acute Kidney Injury in Patients Hospitalized with Pneumonia: A Prospective Observational Study
}

\author{
Muzamil Latief ${ }^{1}$, Reyaz Ahmed Para ${ }^{2}$, Obeid Shafi ${ }^{3}$, Zhahid Hassan $^{4}$, Summyia Farooq ${ }^{5}$, Farhat Abbas ${ }^{5}$ (D) \\ Received: 10 Feb 2021 \\ Published: 10 Nov 2021
}

\begin{abstract}
Background: Acute kidney injury (AKI) is frequent in hospitalized patients with critical illness and presents in up to one-quarter of patients with non-severe community-acquired pneumonia (CAP), resulting in increased short and long-term mortality. There is a paucity of literature from resource-limited settings regarding the incidence and risk factors for AKI in patients with CAP. In this study, we looked at the incidence and risk factors for AKI in patients hospitalized with CAP in a resource-limited setting

Methods: This prospective observational study conducted over 1 year period included patients $\geq 18$ years of age diagnosed with CAP admitted to a tertiary care center. The differences in baseline characteristics between hospitalized ICAP patients with and without AKI; and risk factors for AKI and the need for renal replacement therapy (RRT) were analyzed using Chi-square test, t-test, MannWhitney $U$ test, and logistic regression with p-value $<0.05$ considered statistically significant.

Results: We observed $27.6 \%(58 / 210)$ of patients had AKI in our study. Patients with AKI had significantly higher baseline comorbidities of chronic kidney disease $(\mathrm{p}=0.005)$ and coronary artery disease $(\mathrm{p}=0.032)$, and significantly higher uric acid $(\mathrm{p}=0.002)$, lower albumin $(\mathrm{p}=0.005)$, lower total protein $(\mathrm{p}=0.015)$, higher bilirubin $(\mathrm{p}=0.001)$, higher LDH $(\mathrm{p}=0.041)$, and higher CURB-65 score $(\mathrm{p}<0.001)$ in addition to elevated creatinine, BUN $(\mathrm{p}<0.001)$ compared to the no-AKI group. The patient group requiring RRT had significantly more males $(\mathrm{p}=0.019)$, with significantly higher phosphorus $(\mathrm{p}=0.038)$, lower ALT $(\mathrm{p}=0.022)$, and expectedly higher creatinine $(\mathrm{p}<0.001)$ and blood urea nitrogen $(\mathrm{p}=0.016)$. The adjusted logistic regression analysis revealed that patients with higher CURB-65 scores were at increased odds of undergoing RRT (OR 8.74, 95\% CI 5.27 to 12.21, $\mathrm{p}=0.039$ ).
\end{abstract}

Conclusion: There is a high incidence of AKI in patients hospitalized for CAP in developing countries. Clinicians should be alert for the prevention and early detection of AKI in CAP patients.

Keywords: Kidney Injury, Pneumonia, Dialysis, CURB-65

Conflicts of Interest: None declared

Funding: None

*This work has been published under CC BY-NC-SA 1.0 license.

Copyright $\odot$ Iran University of Medical Sciences

Cite this article as: Latief M, Ahmed Para R, Shafi O, Hassan Z, Farooq S, Abbas F. Incidence and Risk Factors of Acute Kidney Injury in Patients Hospitalized with Pneumonia: A Prospective Observational Study. Med J Islam Repub Iran. 2021 (10 Nov);35:150. https://doi.org/10.47176/mjiri.35.150

\section{Introduction}

Acute Kidney Injury (AKI, previously acute renal failure) is a clinical syndrome of rapid onset renal impairment, with a decrease in excretory function and accumulation of products of nitrogen metabolism (including urea, creatinine, and other unmeasured product s) $(1,2)$. AKI has been reported to be present in up to $22 \%$ of hospital-

\footnotetext{
Corresponding author: Dr Farhat Abbas, farahabbas.m@gmail.com

1. Department of Nephrology, Government Medical College, Srinagar, Kashmir, India

2. Department of Emergency Medicine, SKIMS, Srinagar, Kashmir, India

3. Flushing Hospital Medical Center, New York, USA

4. Department of Medicine, Government Medical College, Baramula, Kashmir, India

5. Pathology Division, Government Medical College, Srinagar, Kashmir, India
}

ized adults, with a much higher incidence (40-60\%) reported in critically ill adults (3). There are multiple definitions used for AKI, with the criteria proposed by the Acute Kidney Injury Network (AKIN) group in 2007 widely accepted (2). An acute decrease in kidney function is classified as AKI if there is an absolute increase in se-

\section{$\uparrow$ What is "already known" in this topic:}

Community-acquired pneumonia leads to Acute Kidney Injury (AKI) through various mechanisms. AKI increases hospital stay, morbidity and mortality.

$\rightarrow$ What this article adds:

As there are limited data evaluating the incidence and risk factors for $\mathrm{AKI}$ in patients of community-acquired pneumonia from resource-limited countries, our study is an effort to bridge this knowledge gap. 
rum creatinine of $\geq 0.3 \mathrm{mg} / \mathrm{dL}$, an increase in serum creatinine of $\geq 50 \%$ (1.5-fold from baseline), or a reduction in urine output $(<0.5 \mathrm{ml} / \mathrm{kg}$ per hour for $>6$ hours). AKI in hospitalized patients is an independent adverse prognostic factor, with a reported mortality of $24 \%$, and associated with more than twice the twice mortality rate in critically ill patients without AKI (3). Various risk factors associated with hospital-acquired AKI (HAAKI) include infection, sepsis, use of nephrotoxic medications, intravenous contrast administration, major surgeries, and underlying kidney disease $(1,4)$. Community-acquired pneumonia (CAP) is one of the most common reasons for hospital admission, and previous studies have suggested that the development of AKI during hospitalization for pneumonia substantially increases mortality $(5,6)$. The mechanism of development of AKI in these patients is presumed to be multifactorial, with inflammation induced by an infectious process thought to play a prominent role $(5,7)$. The proinflammatory cytokines could cause or exacerbate impaired renal function by direct renal endothelial injury, vasoplegia, altered mitochondrial function, direct tubular injury, or causing cell cycle arrest in addition to other mechanisms (8-12). AKI is not uncommon even in patients with non-severe CAP (up to $25 \%$ of patients), resulting in increased short- and long-term mortality $(1,7)$.

There are limited data evaluating the incidence and risk factors for AKI in patients hospitalized with CAP and we could not identify any studies from resource-limited countries looking at this subset of patients in our literature review. We examined the incidence and risk factors for AKI in patients hospitalized with CAP at a tertiary care hospital in India.

\section{Methods}

This prospective observational study conducted over 1 year period included 210 patients. Patients $\geq 18$ years were included in the study if they had three or more of the following symptoms or signs: fever, new-onset cough with or without sputum production, pleuritic chest pain, dyspnea, or altered breath sound on auscultation, plus a chest radiograph showing a new pulmonary infiltrate compatible with the presence of acute pneumonia (13). Exclusion criteria included hospital-acquired pneumonia (development of symptoms 48 hours following admission); or active thoracic malignancy. Demographic data were collected, and past history was noted for any underlying comorbid illnesses such as diabetes mellitus, chronic obstructive pulmonary disease (COPD), chronic kidney disease (CKD), coronary artery disease (CAD), chronic liver disease (CLD), or hypertension (HTN). The study was approved by the Institutional Ethics Committee and all the participants consented to the study. The severity of pneumonia at presentation was assessed by the CURB-65 score (14). All the patients underwent routine investigations in the form of complete blood count, routine serum biochemistry, radiography of the chest, electrocardiogram, and computed tomography of the chest when indicated. The patients were managed as per the protocol of the treating unit. We classified patients with acute kidney injury if they met the criteria at any time during hospitali- zation as per Acute kidney injury network (AKIN) criteria. In patients with no history of CKD or pre-hospital creatinine value, we estimated premorbid creatinine using the Modification of Diet in Renal Disease (MDRD) equation and subsequently selected the lower creatinine value from either the MDRD creatinine or hospital admission creatinine as the baseline value (15). Differences in baseline characteristics between hospitalized CAP patients with and without AKI were evaluated. We also assessed laboratory predictors for the development of AKI and the requirement for renal replacement therapy. The decision to proceed to renal replacement therapy (RRT) was at the discretion of the treating unit.

\section{Statistical Analyses}

The results were analyzed using SPSS statistical software version 19.0 (IBM Corporation; Armonk, NY, USA). After analyzing the data for distribution and skewness, descriptive statistics were applied and discrete variables were analyzed using the Chi-square test for categorical data. Normally distributed continuous variables were compared using Welch's t-test for independent samples, while variables showing non-Gaussian distribution were subjected to the Mann-Whitney U test. Variables were subjected to binary logistic regression to examine the association with AKI, using odds ratios (OR). We considered $p$-value $<0.05$ as statistically significant.

\section{Results}

We analyzed 210 patients with CAP who met the study inclusion criteria. There was a slight male preponderance $(\mathrm{n}=110,52.3 \%)$, with the median age of the study population being 59 years (Range 18 to 95 years, $\mathrm{SD}=15.6$ ). A total of $58(27.6 \%)$ patients developed AKI. The demographic characteristics and comorbidities for patients in AKI and no-AKI groups are compared in Table 1. Patients in the AKI group had a significcantly higher proportion of patients with co-morbidities like CKD $(p=0.005)$ and CAD $(\mathrm{p}=0.032)$.

A comparison of laboratory characteristics revealed significantly higher uric acid $(\mathrm{p}=0.002)$, lower albumin levels $(\mathrm{p}=0.005)$, lower total protein $(\mathrm{p}=0.015)$, higher bilirubin $(p=0.001)$, and higher LDH levels $(p=0.041)$ in the AKI group in addition to the elevated creatinine, BUN $(\mathrm{p}<0.001)$ compared to the no-AKI group (Table 2$)$. We observed that the CURB-65 score was higher in the AKI compared to the non-AKI group $(\mathrm{p}<0.001)$.

The association of AKI with uric acid (OR 1.101, 95\% CI 0.97 to $1.24, \mathrm{p}=0.134$ ), albumin (OR $0.629,95 \% \mathrm{CI}$ 0.29 to $1.32, \mathrm{p}=0.221$ ), total protein (OR $0.809,95 \% \mathrm{CI}$ 0.48 to $1.35, \mathrm{p}=0.421$ ), and bilirubin (OR $1.722,95 \% \mathrm{CI}$ 0.96 to $3.07, p=0.066$ ) levels were not statistically significant in binary logistic regression analysis, after adjusting for creatinine and BUN levels. Increased LDH levels minimally increased odds for AKI (OR 1.001, 95\% CI 1.0001 to $1.002, \mathrm{p}=0.036$ ), while higher CURB-65 score (OR $1.794,95 \%$ CI 1.25 to $2.55, \mathrm{p}=0.001)$ continued to be significantly associated with AKI (Table 3 ).

Out of the 58 patients who developed AKI, 8 (13.8\%) needed RRT. All the patients needing RRT were male 
Table 1. Baseline characteristics in AKI and no-AKI groups (* SD: Standard deviation, SBP: Systolic blood presisure, DBP: Diastolic blood pressure, COPD: Chronic obstructive pulmonary disease, CKD: Chronic kidney disease, CAD: Coronary artery disease, CLD: Chronic liver disease)

\begin{tabular}{|c|c|c|c|}
\hline Variable & AKI $(n=58)$ & No-AKI $(n=152)$ & p-value \\
\hline Age in years, Mean (SD) & $60.2(13.2)$ & $58(16.4)$ & 0.356 \\
\hline Gender, $\mathrm{n}(\%)$ & & & 0.091 \\
\hline Male & $36(17.1)$ & $74(35.2)$ & \\
\hline Female & $22(10.5)$ & $78(37.2)$ & \\
\hline SBP in mmHg, Mean (SD) & $108(17.4)$ & $113.2(18)$ & 0.062 \\
\hline DBP in $\mathrm{mmHg}$, Mean (SD) & $70.2(11.7)$ & $71.3(11)$ & 0.518 \\
\hline \multicolumn{4}{|l|}{ COPD (n) } \\
\hline Yes & 21 & 58 & 0.874 \\
\hline No & 37 & 94 & \\
\hline \multicolumn{4}{|l|}{ CKD (n) } \\
\hline Yes & 13 & 12 & 0.005 \\
\hline No & 45 & 140 & \\
\hline \multicolumn{4}{|l|}{ CAD (n) } \\
\hline Yes & 9 & 9 & 0.032 \\
\hline No & 49 & 143 & \\
\hline \multicolumn{4}{|l|}{ CLD (n) } \\
\hline Yes & 1 & 2 & 1.000 \\
\hline No & 57 & 150 & \\
\hline \multicolumn{4}{|l|}{ Hypertension (n) } \\
\hline Yes & 33 & 79 & 0.540 \\
\hline No & 25 & 73 & \\
\hline
\end{tabular}

Table 2. Laboratory values for AKI and no-AKI groups

\begin{tabular}{|c|c|c|c|}
\hline Variable & AKI $(n=58)$ & No-AKI $(n=15.2)$ & p-value \\
\hline Creatinine in $\mathrm{mg} / \mathrm{dL}$, Mean (SD) & $2.77(1.71)$ & $1.12(0.35)$ & $<0.001$ \\
\hline BUN in $\mathrm{mg} / \mathrm{dL}$, Mean (SD) & $104.5(69.9)$ & $51.0(23.3)$ & $<0.001$ \\
\hline Calcium in $\mathrm{mg} / \mathrm{dL}$, Mean (SD) & $8.8(0.8)$ & $9.0(0.7)$ & 0.094 \\
\hline Uric acid in $\mathrm{mg} / \mathrm{dL}$, Mean (SD) & $6.1(2.7)$ & $4.8(2.4)$ & 0.002 \\
\hline Albumin in g/dL, Mean (SD) & $3.04(0.51)$ & $3.28(0.57)$ & 0.005 \\
\hline Phosphorus in mg/dL, Mean (SD) & $11.2(41.3)$ & $3.0(0.8)$ & 0.137 \\
\hline Total Bilirubin in mg/dL, Mean (SD) & $1.2(0.7)$ & $0.9(0.5)$ & 0.001 \\
\hline Total Protein in g/dL, Mean (SD) & $5.9(0.7)$ & $6.2(0.7)$ & 0.015 \\
\hline ALT/SGPT in IU/L, Mean (SD) & $88.1(111.9)$ & $58.7(67.7)$ & 0.067 \\
\hline LDH in IU/L, Mean (SD) & $492.8(492.0)$ & $353.9(205.4)$ & 0.041 \\
\hline Hemoglobin in g/dL, Mean (SD) & $11.1(2.4)$ & $11.8(2.3)$ & 0.051 \\
\hline WBC count $\times 10^{9} / \mathrm{L}$, Mean (SD) & $12.1(11)$ & $10.7(4.3)$ & 0.180 \\
\hline CURB-65 score & $2.3(0.9)$ & $1.6(1)$ & $<0.001$ \\
\hline \multicolumn{4}{|l|}{ ICU admission (n) } \\
\hline Yes & 4 & 17 & 0.447 \\
\hline No & 54 & 135 & \\
\hline \multicolumn{4}{|l|}{ Mortality (n) } \\
\hline No & 55 & 142 & 0.494 \\
\hline Yes & 3 & 10 & \\
\hline
\end{tabular}

Table 3. Results of regression analysis of biochemical parameters and CURB 65 score vis a vis AKI

\begin{tabular}{|c|c|c|c|c|c|c|}
\hline Variable & Beta coefficient & SE (beta coefficient) & Lower limit of CI & Upper limit of CI & Odds Ratio & p-value \\
\hline Uric acid & 0.0965 & 0.0645 & 0.970 & 1.249 & 1.101 & 0.134 \\
\hline Albumin & -0.4632 & 0.3791 & 0.299 & 1.322 & 0.629 & 0.221 \\
\hline Bilirubin & 0.5436 & 0.2958 & 0.964 & 3.075 & 1.722 & 0.066 \\
\hline Total protein & -0.2110 & 0.2623 & 0.484 & 1.354 & 0.809 & 0.421 \\
\hline LDH & 0.0013 & 0.0006 & 1.0001 & 1.002 & 1.001 & 0.036 \\
\hline CURB-65 score & 0.5847 & 0.1808 & 1.259 & 2.557 & 1.794 & 0.001 \\
\hline
\end{tabular}

(Table 4). We also analyzed the data for laboratory values associated with RRT in patients with AKI. As expected, the RRT group had significantly higher creatinine $(\mathrm{p}<0.001)$ and blood urea nitrogen $(\mathrm{p}=0.016)$. The number of male patients was significantly higher in the RRT group compared to the no-RRT group $(\mathrm{p}=0.019)$. Also, patients in the RRT group were observed to have significantly higher phosphorus $(p=0.038)$, and lower ALT $(p=0.022)$ levels. Although the CURB-65 score showed a trend for higher scores in the RRT group, the difference did not attain statistical significance $(\mathrm{p}=0.064)$. In the regression analysis, ALT (OR $0.98,95 \%$ CI 0.97 to $0.99, \mathrm{p}=0.031$ ) levels were marginally associated with a need for RRT, while phosphorus levels did not show a statistically significant association (OR 5.5, 95\% CI 0.85 to 10.14 , $\mathrm{p}=0.083$ ). Pertinently, the adjusted logistic regression analysis revealed that patients with higher CURB-65 scores were at higher odds of undergoing RRT (OR 8.74, $95 \%$ CI 5.27 to $12.21, \mathrm{p}=0.039$ ).

\section{Discussion}

We report an incidence of AKI in $27.6 \%$ of patients hospitalized for CAP in our study. Factors associated with AKI in these patients include past history of CKD or 
Table 4. Comparison of patients requiring RRT with no-RRT group

\begin{tabular}{|c|c|c|c|}
\hline Variable & $\operatorname{RRT}(n=8)$ & No-RRT $(n=50)$ & p-value \\
\hline Age in years, Mean (SD) & $63.4(16.5)$ & $59.7(12.7)$ & 0.560 \\
\hline \multicolumn{4}{|l|}{ Gender $(\mathrm{n})$} \\
\hline Male & 8 & 28 & 0.019 \\
\hline Female & 0 & 22 & \\
\hline Creatinine in $\mathrm{mg} / \mathrm{dL}$, Mean (SD) & $6.37(2.06)$ & $2.18(0.63)$ & $<0.001$ \\
\hline BUN in $\mathrm{mg} / \mathrm{dL}$, Mean (SD) & $216.3(116.3)$ & $86.6(37.2)$ & 0.016 \\
\hline Calcium in $\mathrm{mg} / \mathrm{dL}$, Mean (SD) & $8.7(0.8)$ & $8.8(0.8)$ & 0.813 \\
\hline Uric acid in $\mathrm{mg} / \mathrm{dL}$, Mean (SD) & $8.1(4.3)$ & $5.8(2.3)$ & 0.255 \\
\hline Albumin in $\mathrm{g} / \mathrm{dL}$, Mean (SD) & $3.1(0.5)$ & $3.0(0.5)$ & 0.908 \\
\hline Phosphorus in mg/dL, Mean (SD) & $5.1(2.1)$ & $3.2(0.9)$ & 0.038 \\
\hline Total Bilirubin in mg/dL, Mean (SD) & $2.05(1.2)$ & $1.17(0.5)$ & 0.083 \\
\hline Total Protein in g/dL, Mean (SD) & $6.3(0.5)$ & $5.9(0.7)$ & 0.09 \\
\hline ALT/SGPT in IU/L, Mean (SD) & $42.6(39.6)$ & $95.5(118.2)$ & 0.022 \\
\hline $\mathrm{LDH}$ in IU/L, Mean (SD) & $340(150.3)$ & $517.3(523)$ & 0.477 \\
\hline Hemoglobin in g/dL, Mean (SD) & $10.83(2.62)$ & $11.17(2.43)$ & 0.742 \\
\hline WBC count $\times 10^{9} / \mathrm{L}$, Mean (SD) & $13.72(2.65)$ & $11.95(11.82)$ & 0.359 \\
\hline CURB-65 score & $3.0(0.9)$ & $2.2(0.9)$ & 0.064 \\
\hline
\end{tabular}

CAD, certain laboratory parameters (high uric acid, low albumin, low total protein, high bilirubin, and high LDH levels), and higher CURB-65 score (i.e., more severe pneumonia). However, the laboratory parameters of uric acid, albumin, total protein, and bilirubin levels were not seen to increase the odds for AKI independently in the regression analysis, and the relation for $\mathrm{LDH}$ levels was also attenuated in the adjusted analysis (a unit increase in LDH levels increases odds of AKI by $0.1 \%$ ). Pneumonia severity was significantly associated with AKI independently, with an increase of CURB-65 score by a single digit resulting in $79.4 \%$ higher odds of AKI.

Although it is difficult to know the exact incidence of AKI in developing countries due to the paucity of data, it is estimated that the burden of AKI is quite high as it is all over the world. A meta-analysis has suggested a pooled incidence of HAAKI between 7.5- 31\% and a mortality of $14-37 \%$ in different parts of Asia $(3,16)$. Most of the data regarding the epidemiology of AKI in India is from single-center studies, with wide fluctuations in incidence and causes of AKI in hospitalized or critically ill patients (17, 18). An incidence of AKI in 6-46\% of critically ill patients has been reported in various studies, with sepsis, tropical illnesses (like malaria, scrub typhus, dengue), pneumonia, pyelonephritis, H1N1 influenza, viral hepatitis, leptospirosis, undifferentiated febrile illness, enteric fever, toxins and envenomation as some of the causes reported with widely fluctuating frequencies $(4,17-21)$. Pneumonia was reported as the most common medical illness in sepsisassociated AKI in a study from Nepal, documented in $50 \%$ of the study population with AKI in the intensive care unit (ICU) (21). Viral pneumonia are also associated with high rates of AKI. During the H1N1 pandemic of 2009, AKI was seen as a frequent complication in critically ill patients with incidence rates of $30-50 \%(22,23)$. Similarly, AKI is now recognized as a well-known complication of the novel COVID-19 illness. A recent report observed an incidence of $>20 \%$ in hospitalized patients, increasing to $>50 \%$ in ICU patients, causing adverse outcomes with the potential for overburdening already stretched healthcare resources (24).

The observed incidence of AKI in patients with CAP in our study was similar to that noted in previous Western studies $(6,7)$. Akram et al. observed around $18 \%$ of patients with CAP have AKI at admission in their study (6). Murugram et al. reported an overall $34 \%$ incidence of AKI in patients with CAP, with up to a quarter of nonsevere pneumonia patients also developing AKI (7). Also, the higher percentage of AKI seen in severe CAP agrees with our study finding showing AKI is associated with higher CURB-65 scores $(6,7)$. The risk factors associated with AKI in our study included comorbidities like CKD or CAD, which have been reported previously $(6,7,25)$. We also observed laboratory features of higher uric acid, lower albumin and total protein, higher bilirubin, and higher LDH levels in patients with AKI. In their study of elderly patients with AKI, Sevor et al. (26) observed AKI more frequently with altered sensorium and abnormal liver function tests (including hyperbilirubinemia). The incidence of AKI in patients was noted to be higher in males in patients with pneumococcal pneumonia (27). Although we did not note this association for the incidence of AKI, the need for RRT in patients with AKI was significantly higher in males in our study. Also, we observed that around $3.8 \%(n=8)$ of the study population ended up requiring RRT during the hospital stay. This is in close agreement with a previous study, where $2.4 \%$ of patients admitted with CAP required RRT (6).

This study, the first of its kind in a resource-limited setting, highlights the high incidence of AKI in patients hospitalized for CAP, similar to that reported from resourcerich countries. It is recognized that the lower incidence of HAAKI reported from the developing nations could potentially be a result of the under-recognition of AKI due to a lack of awareness among health care providers and limited available resources $(28,29)$. It cannot be overemphasized that clinicians need to be alert for the prevention and early detection of AKI in patients admitted with this common diagnosis. A few strategies suggested include frequent risk assessment for hospitalized patients (at admission and during stay); optimizing fluid volume, blood pressure and perfusion; correction and maintenance of electrolyte, acid-base, and mineral homeostasis; prompt treatment of hypotension and sepsis; avoiding the use of nephrotoxic drugs especially in those with or at risk of $\mathrm{AKI}$; and if needed they should be administered in dosage 
based on the volume of distribution in the elderly, obese and malnourished patients; and drug level monitoring for nephrotoxic medications $(29,30)$.

\section{Study Limitations}

This was a single-center observational study. As is common with studies of AKI, values of baseline creatinine values were not available for all patients and were estimated based on MDRD guidelines, which is an established methodology. Since the etiopathogenesis of AKI is often multifactorial, confounding factors like patient medications, fluids, and hemodynamic status could have influenced the development of AKI and its course. Future studies could focus on the role of these confounding factors, especially the administration of nephrotoxic medications. Furthermore, we did not have a long-term follow-up for the development of residual kidney disease or other complications.

\section{Conclusion}

There is a high incidence of AKI in patients hospitalized for CAP in developing countries, similar to that reported from resource-rich countries. Clinicians should be alert for prevention and early detection of AKI in patients admitted with this common diagnosis. Further studies should be conducted to investigate long-term complications from $\mathrm{AKI}$ in these patients and evaluate strategies for prevention and treatment in resource-limited settings

\section{Acknowledgments}

We would like to thank our patients, dialysis technicians, and Dr. Imran Shafi for his help.

\section{Conflict of Interests}

The authors declare that they have no competing interests.

\section{References}

1. Bellomo R, Kellum JA, Ronco C. Acute kidney injury. Lancet. 2012 Aug 25;380(9843):756-66.

2. Mehta RL, Kellum JA, Shah SV, Molitoris BA, Ronco C, Warnock DG, et al. Acute Kidney Injury Network. Acute Kidney Injury Network: report of an initiative to improve outcomes in acute kidney injury. Crit Care. 2007;11(2):R31

3. Susantitaphong P, Cruz DN, Cerda J, Abulfaraj M, Alqahtani F, Koulouridis I, et al. Acute Kidney Injury Advisory Group of the American Society of Nephrology. World incidence of AKI: a metaanalysis. Clin J Am Soc Nephrol. 2013 Sep;8(9):1482-93.

4. Kaaviya R, Vadivelan M, Balamurugan N, Parameswaran S, Thabah MM. Community Acquired AKI: A Prospective Observational Study from a Tertiary Level Hospital in Southern India. Indian J Nephrol. 2019 Jul-Aug;29(4):254-260..

5. Chawla LS, Amdur RL, Faselis C, Li P, Kimmel PL, Palant CE. Impact of Acute Kidney Injury in Patients Hospitalized With Pneumonia. Crit Care Med. 2017 Apr;45(4):600-606.

6. Akram AR, Singanayagam A, Choudhury G, Mandal P, Chalmers JD, Hill AT. Incidence and prognostic implications of acute kidney injury on admission in patients with community-acquired pneumonia. Chest. 2010 Oct;138(4):825-32.

7. Murugan R, Karajala-Subramanyam V, Lee M, Yende S, Kong L, Carter M, et al. Genetic and Inflammatory Markers of Sepsis (GenIMS) Investigators. Acute kidney injury in non-severe pneumonia is associated with an increased immune response and lower survival. Kidney Int. 2010 Mar;77(6):527-35.

8. Adembri C, Sgambati E, Vitali L, Selmi V, Margheri M, Tani A, et al.
Sepsis induces albuminuria and alterations in the glomerular filtration barrier: a morphofunctional study in the rat. Crit Care. 2011;15(6):R277.

9. Chelazzi C, Villa G, Mancinelli P, De Gaudio AR, Adembri C. Glycocalyx and sepsis-induced alterations in vascular permeability. Crit Care. 2015 Jan 28;19(1):26.

10. Ince $\mathrm{C}$. The central role of renal microcirculatory dysfunction in the pathogenesis of acute kidney injury. Nephron Clin Pract. 2014;127(14):124-8.

11. Zarbock A, Gomez H, Kellum JA. Sepsis-induced acute kidney injury revisited: pathophysiology, prevention and future therapies. CurrOpin Crit Care. 2014 Dec;20(6)):588-95.

12. Parikh SM, Yang Y, He L, Tang C, Zhan M, Dong Z. Mitochondrial function and disturbances in the septic kidney. Semin Nephrol. 2015 Jan;35(1):108-19.

13. Mandell LA, Wunderink RG, Anzueto A, Bartlett JG, Campbell GD, Dean NC, et al. Infectious Diseases Society of America; American Thoracic Society. Infectious Diseases Society of America/American Thoracic Society consensus guidelines on the management of community-acquired pneumonia in adults. Clin Infect Dis. 2007 Mar 1;44 Suppl 2(Suppl 2):S27-72.

14. Lim WS, van der Eerden MM, Laing R, Boersma WG, Karalus N, Town GI, et al. Defining community acquired pneumonia severity on presentation to hospital: an international derivation and validation study. Thorax. 2003 May;58(5):377-82.

15. National Kidney Foundation. K/DOQI clinical practice guidelines for chronic kidney disease: evaluation, classification, and stratification. Am J Kidney Dis. 20012 Feb;39(2 Suppl 1):S1-266.

16. Yang L. Acute Kidney Injury in Asia. Kidney Dis (Basel). 2016 Oct;2(3):95-102.

17. Kulkarni AP, Bhosale SJ. Epidemiology and Pathogenesis of Acute Kidney Injury in the Critically Ill Patients. Indian J Crit Care Med. 2020 Apr;24(Suppl 3):S84-S89.

18. Priyamvada PS, Jayasurya R, Shankar V, Parameswaran S. Epidemiology and Outcomes of Acute Kidney Injury in Critically Ill: Experience from a Tertiary Care Center. Indian J Nephrol. 2018 NovDec;28(6):413-420.

19. Vikrant S, Gupta D, Singh M. Epidemiology and outcome of acute kidney injury from a tertiary care hospital in India. Saudi J Kidney Dis Transpl. 2018 Jul-Aug;29(4):956-966.

20. Mehta K, Pajai A, Bhurke S, Shirkande A, Bhadade R, D'Souza R. Acute Kidney Injury of Infectious Etiology in Monsoon Season: A Prospective Study Using Acute Kidney Injury Network Criteria. Indian J Nephrol. 2018 Mar-Apr;28(2):143-152.

21. Ghimire M, Pahari B, Sharma SK, Thapa L, Das G, Das GC. Outcome of sepsis-associated acute kidney injury in an intensive care unit: an experience from a tertiary care center of central Nepal. Saudi J Kidney Dis Transpl. 2014 Jul;25(4):912-7.

22. Martin-Loeches I, Papiol E, Rodríguez A, Diaz E, Zaragoza R, Granada RM, et al. H1N1 SEMICYUC Working Group. Acute kidney injury in critical ill patients affected by influenza A (H1N1) virus infection. Crit Care. 2011 Feb 22;15i(1):R66.

23. Pettilä V, Webb SA, Bailey M, Howe B, Seppelt IM, Bellomo R. Acute kidney injury in patients with influenza A (H1N1) 2009. Intensive Care Med. 2011 May;37(5):763-7.

24. Nadim MK, Forni LG, Mehta RL, Connor MJ Jr, Liu KD, Ostermann M, et al. COVID-19-associated acute kidney injury: consensus report of the 25th Acute Disease Quality Initiative (ADQI) Workgroup. Nat Rev Nephrol. 2020 Dec;16(12):747-764.

25. Onk OA, Onk D, Ozcelik F, Gunay M, Turkmen K. Risk Factors for Acute Kidney Injury after Coronary Artery Bypass Surgery and Its Detection Using Neutrophil Gelatinase-Associated Lipocalin Cardiorenal Med. 2016 May;6(3):216-29.

26. Serov VA, Shutov AM, Kuzovenkova MY, Serova DV. (Clinical features of community-acquired pneumonia associated with acute kidney injury in elderly patients.). Adv Gerontol. 2019;32(4):633-638. (Russian)

27. Lin TY, Chen YG, Lin CL, Kao CH. Increased Risk of Acute Kidney Injury following Pneumococcal Pneumonia: A Nationwide Cohort Study. PLoS One. 2016 Jun 30;11(6):e0158501.

28. Mehta RL, Burdmann EA, Cerdá J, Feehally J, Finkelstein F, GarcíaGarcía G, et al. Recognition and mamagement of acute kidney injury in the International Society of Nephrology 0by 25 Global Snapshot: a multinational cross-sectional study. Lancet. 2016 May 14;387(10032):2017-25. 
AKI in Pneumonia

29. Kher V, Srisawat N, Noiri E, BenghanemGharbi M, Shetty MS, Yang L, et al. Acute Disease Quality Initiative (ADQI) Consensus Group. Prevention and Therapy of Acute Kidney Injury in the Developing World. Kidney Int Rep. 2017 Apr 26;2(4):544-58.

30. Yamout H, Levin ML, Rosa RM, Myrie K, Westergaard S. Physician

Prevention of Acute Kidney Injury. Am J Med. 2015 Sep;128(9):1001-6. 